\title{
ESTADISTICA
}

\section{Desequilibrio entre obligaciones y recursos en el Municipio rural}

\section{(Apostillas a un libro reciente)}

El Instituto de Estudios de Administración Local ha publicado un libro, del que es autor el Sr. Paredes Marcos, sobre el tema que encabeza este artículo. En otro lugar de este mismo número encontrará el lector la referencia bibliográfica correspondiente.

Como se trata de un libro en el que por primera vez en España (por lo menos que sepamos rosotros) se desarrolla un tema municipalista sobre bases estadísticas rigurosas, hemos creído que el suceso debería registrarse en esta sección, no por lo que tiene de suceso bibliográfico-científico, sino por lo que tiene de suceso estadístico-municipalista. $Y$, naturalmente, registrarlo significa para nosotros, no hacer el elogio del autor y del libro (hecho ya suficientemente al consignar que uno y otro han merecido el «Premio Calvo Sotelo)), sino examinar y aun confrontar con nuestro propio criterio la tesis mantenida por el autor y el aparato estadístico (documentación y métodos) de que se ha valido para sostenerla.

\section{II}

En la obra, primeramenté, se suscita una cuestión de especial interés para el que esto escribe; nos referimos a lo que debe entenderse por Municipio rural; dicho de otro modo, ¿cuándo se puede considerar que un Municipio deja de ser rural para tomar caracte- 
res urbanos? ¿Es suficiente un determinado volumen de población? Si así fuera, en Galicia encontrariamos términos municipales habitados por 15 ó 20 mil personas, y que son simples agrupaciones de aldeas; en cambio, en Cataluña y Vascongadas encontraríamos pequeños Municipios de cinco, seis $u$ ocho mil habitantes, con todas las características de ciudad. Tal vez fuera mejor tomar como criterio la agrupación o diseminación del caserio. Acaso mejor aún las profesiones $\mathbf{u}$ ocupaciones de la mayoría de sus habitantes.

La cuestión viene tratada en la introducción de una manera tangencial, puesto que el autor lo único que se propone es adoptar un criterio para su trabajo, seleccionándolo entre los tres criterios que encontró establecidos en los documęntos estadístico-administrativos que hubo de manejar.

Por una parte, en la gran encuesta estadística realizada por el Instituto de Estudios de Adminstración Local y por su Sección de Estadística, se adopta el volumen de población de $\mathbf{1 5 . 0 0 0}$ habitantes como línea divisoria entre Municipios urbanos y rurales.

Pero, en cambio, la Estadística de Presupuestos municipales, que forma y publica la Dirección de Administración Local, marca lineas divisorias en los $\mathbf{1 . 0 0 0}$, los $\mathbf{5 . 0 0 0}$, los $\mathbf{2 0 . 0 0 0}$ y los $\mathbf{1 0 0 . 0 0 0}$ habitantes. $Y$ en el censo de población de 1940 , insinúa en su prólogo, que en los $\mathbf{1 0 . 0 0 0}$ habitantes es donde cabe establecer la separación, porque a partir de esa cifra, por encima y por debajo, parecen advertirse cambios sensibles en la estructura de los Municipios.

El autor, después de lamentar esta disparidad, acepta la divisoria en $\mathbf{1 5 . 0 0 0}$ habitantes, tal vez porque la encuesta citada del Instituto de Estudios de Administración Local va a proporcionarle la parte más importante y más probatoria entre la documentación que ha de manejar. Precisamente en el Prólogo se dice que el libro ha podido escribirse por haber encontrado en aquella encuesta los argumentos decisivos para su tesis.

Delimitando el campo de estudio, incluyendo en él únicamente los Municipios de $\mathbf{1 5 . 0 0 0}$ o menos habitantes, a los cuales aplica el calificativo de rural, resulta un área de observación formada por 9.035 Municipios con $\mathbf{1 5 , 9}$ millones de habitantes; es decir, el $\mathbf{9 7 , 6}$ por 100 de los Municipios españoles y el 61,4 por 100 de nuestra población. 


\section{III}

¿Cómo están atendidos los servicios públicos municipales en los 9.035 Municipios que por contar hasta $\mathbf{1 5 . 0 0 0}$ habitantes tienen según el autor, la consideración, o por lo menos el calificativo, de rurales? Las Estadísticas contenidas en la encuesta del Instituto de Estudios van a decirlo. Veintiún cuadros se insertan en el libro, de los cuales hemos extraido nosotros el siguiente resumen, donde se incluyen exclusivamente las cifras relativas:

Por 100 Ayuntamientos de cada grupo, cuántos:

\begin{tabular}{|c|c|c|c|c|c|}
\hline & 1.000 & $1.001-2.0110$ & $2.001-5.000$ & $5.001-10.000$ & $10.0111-15.000$ \\
\hline Con agua mala.......... & 35 & 36 & 8 & 7 & 6 \\
\hline Con 2 litros por habitante.. & 46 & $6 I$ & $4 \mathrm{I}$ & 41 & 42 \\
\hline Sin fuentes en el casco... & $\triangle 4$ & 33 & 14 & I 1 & 9 \\
\hline Sin red domiciliaria ..... & 94 & 85 & 73 & 61 & 42 \\
\hline Sin baños públicos...... & - & & 97 & 89 & 82 \\
\hline Sin alumbrado.......... & 23 & 12 & 12 & 11 & 3 \\
\hline Sin pavimentacion....... & 83 & 69 & 54 & 37 & 22 \\
\hline Sin alcantarillado. . . . . & 93 & 83 & 69 & 55 & 40 \\
\hline Sin lavadero $\ldots, \ldots \ldots$ & $5^{I}$ & 51 & 48 & $5 \mathrm{I}$ & 39 \\
\hline Sin recngida de basura... & 99 & 45 & 64 & 38 & 22 \\
\hline $\begin{array}{l}\text { Sin matadero.. } \\
\text { Sin cuarteles de Guardia }\end{array}$ & 74 & 48 & 31 & 22 & 9 \\
\hline Civil. $\quad \ldots \ldots \ldots \ldots$ & $9 \mathrm{I}$ & ho & 30 & 19 & 7 \\
\hline Sin telègrafo. ..... . . & 79 & 56 & $3^{0}$ & 22 & 9 \\
\hline Sin teléfono.......... & 98 & 91 & 72 & 42 & 17 \\
\hline Sin servicio de incendios. & 48 & 93 & 84 & 77 & 67 \\
\hline Sin hospitales.. $\ldots \ldots \ldots$ & 98 & 89 & 83 & 75 & 52 \\
\hline Sin casas de socorro . . . & - & & 95 & 81 & 63 \\
\hline Sin asilos $\quad: \quad \ldots \quad \ldots \ldots$ & - & - & 91 & 79 & 62 \\
\hline Sin cementerio municipal.. & 25 & 21 & 25 & 29 & 20 \\
\hline Promedio........ & 70.7 & 61,5 & 54,2 & 44,6 & 32,2 \\
\hline
\end{tabular}

Este cuadro nos dice que en 16 servicios de los 19 sometidos a observación, los casos negativos o de inexistencia del servicio, son tanto más numerosos cuanto menor es la población del Municipo. En efecto, los casos de Municipios con agua mala son 35 por 100 en el grupo de 1.000 y menos habitantes, y de 6 por 100, en el grupo de 10-15 mil; los que carecen de fuentes en el casco son 44 por 100 en el primer grupo, y 9 por 100, en el último; los que no tienen 
sistema de distribución de red domiciliaria son, 94 en el primer grupo, y 42 en el último; y los que carecen de alumbrado son, 23 por 100 entre los más pequeños, y 3 por 100 en los mayores de entre los rurales. Renunciamos a continuar, porque el lector puede comprobar por sí mismo en el cuadro el porcentaje comparado que desee. Sólo aparecen como excepción los Municipios escasos de agua (con menos de dos litros por habitante), que sòn entre $41-46$ por 100 en todos los grupos, excepto en el de 1.001-2.000 habitantes, en el cual son 61 por 100 , sin que de las cifras mismas se desprenda ninguna explicación del caso. En los Municipios sin lavadero, parece mantenerse el porcentaje entre 48-51, y sólo en el último grupo baja a 39. $Y$, finalmente, los que carecen dé cementerio municipal; en ellos, el poncentaje fluctúa de una manera un poco errática, entre 20-29 por 100.

El promedio de promedios que hemos obtenido al final, manifiesia rotundamente la proporción en baja de la inexistencia de servicios municipales conforme aumentan las cifras de población. Aunque tales promedios no constituyan un método estadístico muy riguroso, o si se quiere muy ortodoxo, constituyen, sin embargo, un indicio sintético muy expresivo; ellos nos dicen que entre los Municipios de 1.000 y menos habitantes el 70,7 por 100 carece de los servicios municipales básicos; la proporción de tales municipios carentes o deficitarios es de 61,5 por 100 en el grupo de 1.001-2.000 habitantes; entre los de 2.001-5.000 personas es de 54,2 por 100; los Municipios que podemos llamar (carenciales) se reducen a 44,6 por 100 en los pueblos comprendidos entre 5.001-10.000; y para lo de 10.001-15.000, la proporción es relativamente reducida, cifrándose en 32,2 por 100.

Para apurar el contenido del cuadro comparemos de otra manera el primero y el último grupo de Municipios:

En el primero, los Municipios carenciales se agrupan de este modo : 


\section{Municipios de 1.000 y menos habitantes}

\begin{tabular}{|c|c|}
\hline $\begin{array}{l}\text { Servicios } \\
\text { que faltan }\end{array}$ & $\begin{array}{l}\text { Porcentaje de pueblos } \\
\text { en los que faltan } \\
\text { los servicios }\end{array}$ \\
\hline 6 & $90-99$ \\
\hline I & $80-89$ \\
\hline 2 & $70-79$ \\
\hline$x$ & $50-59$ \\
\hline 2 & $40-49$ \\
\hline 1 & $30-39$ \\
\hline 2 & $20-29$ \\
\hline 16 & 70,7 promedio \\
\hline
\end{tabular}

En los Municipios de 10.001-15.000 habitantes, estos Municipios carenciales se agrupan así:

\begin{tabular}{|c|c|}
\hline $\begin{array}{l}\text { Servicios } \\
\text { que faltan }\end{array}$ & $\begin{array}{l}\text { Porcentaje de pueblos } \\
\text { en los que faltan } \\
\text { los servicios }\end{array}$ \\
\hline & - \\
\hline$\cdot \mathbf{I}$ & $80-89$ \\
\hline 3 & $60-69$ \\
\hline 1 & $50-59$ \\
\hline 3 & $40-49$ \\
\hline I & $30-39$ \\
\hline 3 & $20-29$ \\
\hline I & $10-19$ \\
\hline 6 & $0-9$ \\
\hline 19 & 32,2 promedio \\
\hline
\end{tabular}

Comparando los dos cuadros se advierte una cierta simetría invertida que da por resultado un porcentaje de 70,7 Municipios carenciales en el grupo de 1.000 y menos habitantes, y de 32,2 en el grupo de 10.001-15.000. 


\section{IV}

Establecido así con una cierta precisión matemática que la existencia de servicios públicos municipales guarda íntima relación con el volumen de habitantes de cada unidad municipal, o dicho de otra forma, que cuanto más pequeño es un Municipio menos servicios públicos municipales tiene instalados, pasa el autor a analizar los Presupuestos municipales, para poner luego en relación los resultados obtenidos en uno y otro estudio. Hecho nuestro resumen númerico de cifras relativas, se obtiene el siguiente cuadro:

\section{Presupuestos municipales de gastos en 1944}

Porcentaje que representa cáda grupo de capitulos respecto al volumen total de gastos

\begin{tabular}{|c|c|c|c|}
\hline \multirow[t]{2}{*}{. } & \multicolumn{3}{|c|}{ AYUNTAMIENTOS } \\
\hline & De 1.000 & De 1.001-5.000 & De $5.001-20.0 \mathrm{no}$ \\
\hline Obligaciones generales............ & .24 .5 & 23.3 & 22,7 \\
\hline Representación, Recaudación, Personal.. & 28,3 & 24,0 & 21,3 \\
\hline Servicios al vecindario $\ldots \ldots \ldots \ldots$ & $4 \mathrm{I}, 7$ & 48,0 & 51,7 \\
\hline Diversos ....................... & 5,5 & 4,7 & 4,3 \\
\hline & 100,0 & 100,0 & 100,0 \\
\hline
\end{tabular}

Los resultados aqui son aún más concluyentes; los Ayuntamientos muy pequeños sólo pueden dedicar el 41,7 por 100 de sus presipuestos a los servicios públicos de comodidad o beneficio para el vecindario; en cambio los Ayuntamientos de 10.001-15.000 habitantes pueden consagrar a esas atenciones el 51,7 por 100 . Es evidente q'1e aunque no sea más que por este concepto, el vecindario de los peque. ños Municipios se encuentra en inferioridad de condiciones respecto al vecindario de los grandes Municipios rurales.

He aquí ahora el cuadro referente a los Presupuestos de ingreso: 
Presupuestos municipales de ingresos en 1944

Porcentaje que representa cada grupo de capitulos respecto al volumen total de ingresos

\begin{tabular}{|c|c|c|c|}
\hline & \multicolumn{3}{|c|}{ AYONTAMIENTOS } \\
\hline & De 1.000 & De 1.001-5.000 & De $5.001-20.000$ \\
\hline Rentas $y$ bienes $\ldots \ldots \ldots \ldots \ldots \ldots \ldots$ & 28,1 & 15,7 & 5,6 \\
\hline Derechos $y$ tasas $\ldots \ldots \ldots \ldots \ldots \ldots \ldots$ & 4,8 & 12,5 & 23,1 \\
\hline Participación en tributos nacionales..... & 4,3 & 8,2 & 10,1 \\
\hline 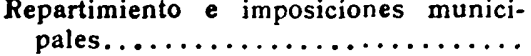 & 52,7 & 49,3 & 40,7 \\
\hline Diversos $\ldots \ldots \ldots \ldots \ldots \ldots \ldots \ldots$ & 10,1 & 14,3 & 20,5 \\
\hline
\end{tabular}

Al tiempo de escribirse este libro, los últimos datos estadísticos sobre Presupuestos municipales eran los de este año de 1944; en el momento presente está publicada ya la información de 1947. Por otra parte, el Decreto de $\mathbf{1 9 4 6}$ ha introducido sensibles modificaciones $\in \mathbf{n}$ las haciendas municipales, las cuales, naturalmente, no aparecen reflejadas en estas cifras de 1944. Hechas estas salvedades, es evidente que el contraste entre los porcentajes de las distintas fuentes de ingreso en los Ayuntamientos pequeños y en los rurales grandes es impresionante; para las Rentas y bienes, la proporción es de 28,1 por 100 en los pequeños, y de 5,6 por 100 en los grandes; y lo mismo ocurre con el Repartimiento (52,7 en los primerosy 40,7 en los últi. mos). En cambio, los Derechos y tasas suben de 4,8 por 100 a $23,1, y$. la participación en tributos nacionales pasa de 4,3 a 10,1 por 100 .

Es curioso hacer resaltar el volumen medio de pesetas del presupuesto de un Ayuntamiento de cada tipo de población y las pesetas por habitante que ese presupuesto medio representa. Esto es lo que hacemos por nuestra propia cuenta, adaptando los datos del libro a la exposición y desarrollo que nosotros vamos haciendo de él:

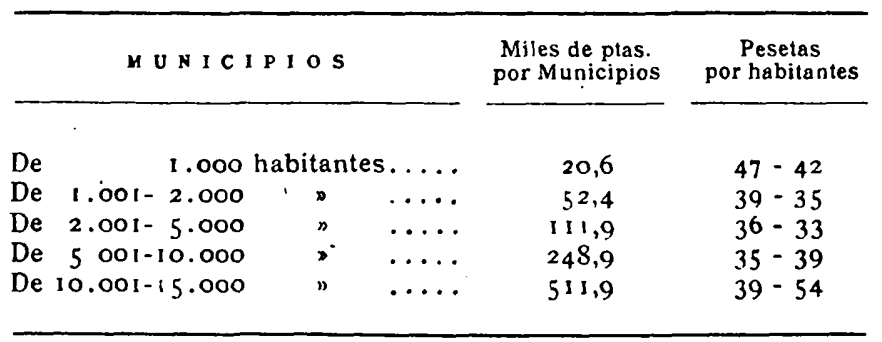


En el cuadro aparece que hasta rebasar el volumen de población de 2.000 habitantes, los Ayuntamientos no rebasan en sus presupuegtos la cifra media de $\mathbf{1 0 0 . 0 0 0}$ pesetas. Ahora bien, es evidente que los presupuestos inferiores a ese volumen minimo han de ser presupuestos carenciales también o insuficientes para el sostenimiento de los servicios públicos mínimos que establece la Ley.

El coste por cabeza marca una ley estadística bien definida. Desde 47 pesetas por habitante que corresponde a los Muncipios mínimos, de menos de $\mathbf{4 0 0}$ habitantes, hasta 33 pesetas por habitante que se produce en los Municipios de 4.000-5.000 habitantes; desde este mínimo vuelve a subir hasta 54 pesetas por habitante en el grupo de 13.00115.000. En el cuadro detallado que aparece en el libro, el coeficiente 35-36 se extiende por una amplia zona central, que comprende 2.494 Municipios con población desde 1.601 hasta 9.000 habitantes. En todo caso, los Municipios pequeños con volúmenes de población comprendidos entre 200 y 1.600 habitantes (6.297 pueblos) pagan de 47 a 39 pesetas por cabeza. En una palabra, expresado el caso en forma paradógica, pero muy gráfica, podríamos decir que los Municipios muy pequeños pagan muy caro el no tener los servicios municipales que exigen las leyes y reclaman las necesidades de la vida moderna.

\section{V}

Una tercera parte de índole estadística trae el libro que nos ocupa. La primera estuvo destinada a demostrar la inexistencia de la mayor parte de los servicios públicos municipales en los Municipios muy pequeños; la segunda, puso de relieve la penuria de dichos Municipios dada la exigüidad de sus presupuestos; la tercera, se propone justifcar la afirmación hecha en la página $\mathbf{1 1 7}$ de que "casi todos los índices de actividad económica $y$, por consiguiente, de bienestar, aumentan conforme el medio ambiente pierde ruralidad y gana en urbanismon. Añadiremos por nuestra cuenta que si bien estamos conformes con el principio, no nos parece la demostración estadistica que hace el autor tan concluyente como en las dos partes anteriores. Véase el cuadro que extractamos del libro: 


\section{Indices diversos de riqueza de los Municipios rurales}

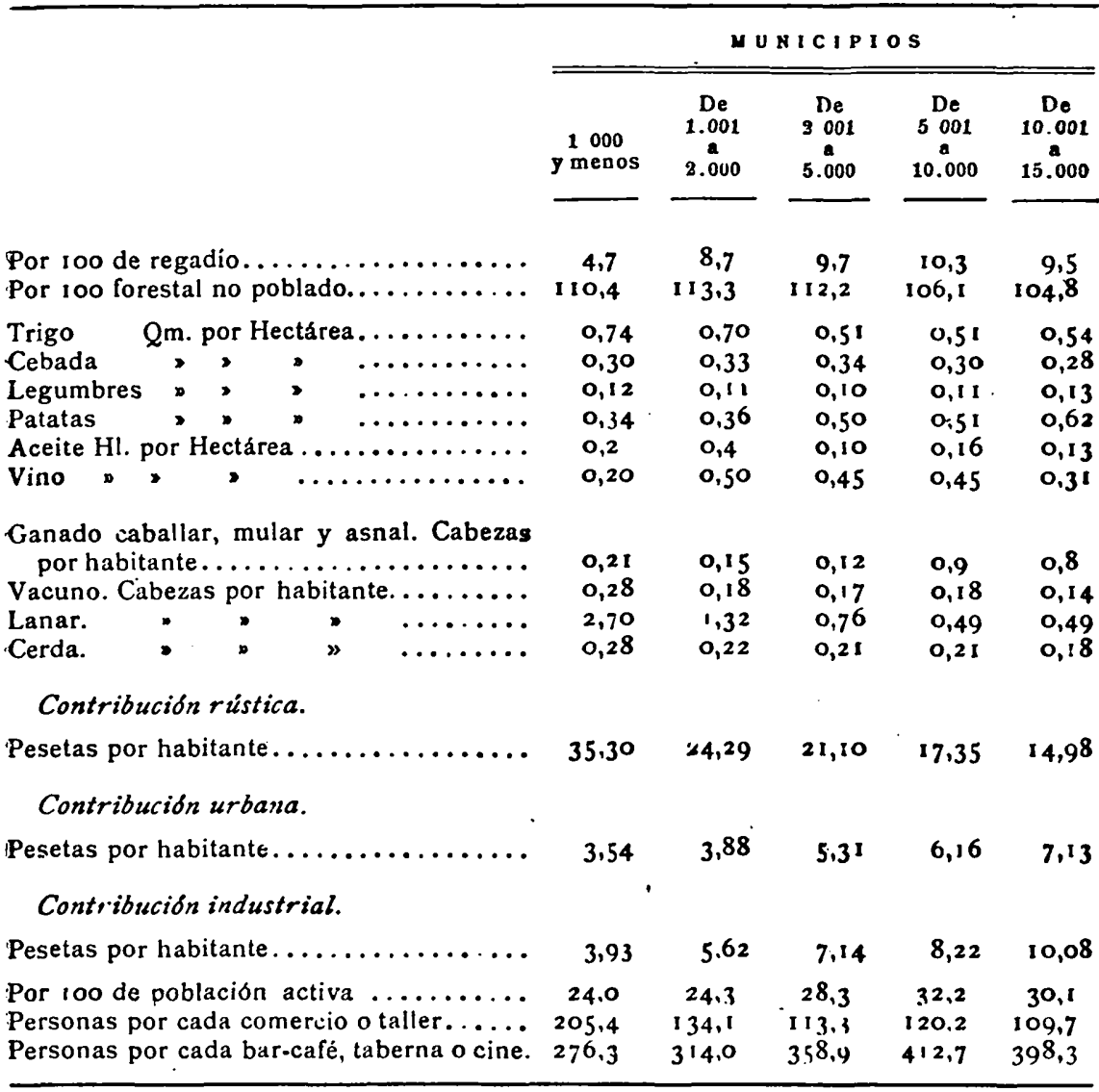

Decimos que no nos parecen tan demostrativas estas cifras como las otras porque en la riqueza de los Municipios, mucho más que en el volumen de su población, influye la sittiación geográfica. La mayor o menor producción y la mayor o menor productividad de un Municipio en vino y aceite, en cereales o patatas, depende de que esté situado en Aragón o en Castilla, en Cataluña o en Andalucía, en Levante o en el Cantábrico; y lo mismo puede decirse de la abundancia o escasez de una especie $\mathfrak{u}$ otra de ganado.

Téngase en cuenta que los datos de producción agricola (lo mismo los absolutos que los relativos) referidos a un solo año, pueden inducir a errores de consideración, mucho más si ese año es el de 1941 - el de 1942, que por su proximidad a los años de guerra puede esiar 
afectado por las anomalías inherentes a los períodos de transición de estados de guerra o estados de paz. El importe mayor o menor de la contribución urbana $o$ industrial y la proporcionalidad de cierta clase de establecimientos, está también más relacionada con la riqueza del término que con el número estricto de pobladores.

A pesar de todo, y aunque la demostración sea deficiente, la conclusión es cierta; y ello es suficiente para el lector.

\section{VI}

No seguiremos al autor en los razonamientos finales del libro. El problema queda planteado en sus términos justos y precisos, y ya sabemos que lo mismo en Matemáticas que en Economía o en Municipalismo, problema bien planteado es problema en breve plazo resuelto. Si así se verifica una vez más en este caso, el autor habrá merecido, además del "Premio Calvo Sotelo», el reconocimiento de todos los hombres de buena voluntad de España.

Nosotros habremos de terminar este artículo manifestando nuestro deseo y nuestra esperanza de que se verifique un acercamiento cara vez mayor entre estadisticos y municipalistas. El libro del señor $\mathrm{Pa}$ redes Marcos ha infundido actualidad, vida e interés en los enormes volúmenes de las frías y secas estadisticas contenidas en la encuesta realizada por el Instituto de Estudios; y, a la vez, ese reservorio ingente de cifras ha proporcionado la cantera de donde el autor que comentamos ha podido encontrar robustos materiales para hacer una construcción modelo de solidez. Ni la Estadística por sí misma, ni el puro análisis doctrinal, hubieran, por sí solos, logrado efectos tan decisivos.

Es preciso tener una estadistica fidedigna y completa de los Municipios españoles; es preciso conocer la estructura intima de nuestros Municipios y las tendencias que siguen en su evolución. La encuesta, tantas veces citada, nos da la pauta ; pero es preciso renovarla, situarla en 1950, y seguir luego, año por año, las transformaciones de cada una de las unidades municipales que la integran. $Y$ con ello los Municipalistas españoles tendrán un instrumento de gran alcance y extrema precisión para sus investigaciones; y los hombres de gobierno podrán actuar sobre el cuerpo vivo de los Municipios españoles, como el médico especialista que emplea una instalación de radiografía antes . de emitir su diagnóstico y de fijar el tratamiento. 
A $\mathrm{E} \times \mathrm{X}$
Cuadros estadísticos detallados de los que se dan resumenes sinteticos
en el texto.

\begin{tabular}{|c|c|c|c|c|c|}
\hline \multirow{2}{*}{$\begin{array}{c}\text { M:U N ICIPIOS } \\
\text { DE : } \\
\text { HABITANTES }\end{array}$} & \multicolumn{5}{|c|}{ Por ioo Municiptos de CADA Catrgoria, cuantos: } \\
\hline & $\underset{\substack{\text { Sin alum } \\
\text { brado }}}{-}$ & Sin pavimen- & $\begin{array}{l}\text { Sin alcantari- } \\
\text { llado }\end{array}$ & $\underset{\text { lavadero }}{\operatorname{Sin}}$ & $\begin{array}{l}\text { Sin recngida } \\
\text { de basuras }\end{array}$ \\
\hline $\begin{array}{rr}- & 400 \\
401- & 600 \\
601- & 800 \\
801-1.000\end{array}$ & $\begin{array}{l}39 \\
20 \\
17 \\
15\end{array}$ & $\begin{array}{l}88 \\
85 \\
82 \\
76\end{array}$ & $\begin{array}{l}97 \\
94 \\
91 \\
89\end{array}$ & $\begin{array}{l}52 \\
51 \\
52 \\
50\end{array}$ & $\begin{array}{l}99,7 \\
99,5 \\
99 \\
98\end{array}$ \\
\hline $\begin{array}{l}1.001-1.200 \\
1.201-1.400 \\
1.40 I=1.600 \\
1.60 I-1.800 \\
1.80 I=2.000\end{array}$ & $\begin{array}{l}12 \\
13 \\
13 \\
12 \\
14\end{array}$ & $\begin{array}{l}74 \\
75 \\
66 \\
66 \\
65\end{array}$ & $\begin{array}{l}86 \\
85 \\
83 \\
81 \\
78\end{array}$ & $\begin{array}{l}50 \\
49 \\
48 \\
49 \\
57\end{array}$ & $\begin{array}{l}97 \\
96 \\
96 \\
91 \\
. \quad 93\end{array}$ \\
\hline $\begin{array}{l}2.001-3.000 \\
3.001-4.006 \\
4.001-5.000\end{array}$ & $\begin{array}{l}12 \\
12 \\
11\end{array}$ & $\begin{array}{l}60 \\
52 \\
50\end{array}$ & $\begin{array}{l}74 \\
65 \\
68\end{array}$ & $\begin{array}{l}50 \\
50 \\
45\end{array}$ & $\begin{array}{l}74 \\
59 \\
59\end{array}$ \\
\hline $\begin{array}{l}5.001-6.000 \\
6.001-7.000 \\
7.001-8.000 \\
8.001-9.000 \\
9.001-10.000\end{array}$ & $\begin{array}{r}13 \\
14 \\
12 \\
10 \\
7\end{array}$ & $\begin{array}{l}48 \\
45 \\
31 \\
33 \\
28\end{array}$ & $\begin{array}{l}68 \\
58 \\
51 \\
54 \\
45\end{array}$ & $\begin{array}{l}50 \\
54 \\
53 \\
43 \\
55\end{array}$ & $\begin{array}{l}54 \\
45 \\
36 \\
33 \\
22\end{array}$ \\
\hline $\begin{array}{l}10.001-11.000 \\
11.001-13.000 \\
13.000-15.000\end{array}$ & $\begin{array}{l}5 \\
3 \\
0\end{array}$ & $\begin{array}{l}24 \\
32 \\
10\end{array}$ & $\begin{array}{l}43 \\
48 \\
29\end{array}$ & $\begin{array}{l}39 \\
38 \\
39\end{array}$ & $\begin{array}{l}22 \\
30 \\
15\end{array}$ \\
\hline
\end{tabular}

Por 100 Municipios de cada categoria, cuantos:

\begin{tabular}{|c|c|c|c|c|}
\hline $\begin{array}{c}\text { MUNICIPIOS } \\
\text { DE: } \\
\text { HABITANTES }\end{array}$ & $\begin{array}{l}\text { Sin } \\
\text { hospitales }\end{array}$ & $\begin{array}{l}\text { Sin cașas } \\
\text { de socorro }\end{array}$ & $\underset{\text { asilos }}{\operatorname{Sin}}$ & $\begin{array}{c}\text { Sin } \\
\text { comenterio } \\
\text { municipal }\end{array}$ \\
\hline 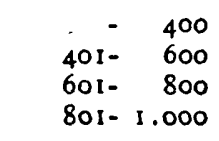 & $\begin{array}{l}99 \\
98 \\
97 \\
97\end{array}$ & . & & $\begin{array}{l}27 \\
28 \\
25 \\
21\end{array}$ \\
\hline $1.001-1.200$ & 95 & & & 21 \\
\hline $\begin{array}{r}1.201-1.400 \\
1.401-1.600 \\
1.601-1.800 \\
1.801-2.000\end{array}$ & $\begin{array}{l}91 \\
87 \\
87 \\
84\end{array}$ & & . & $\begin{array}{l}23 \\
17 \\
25 \\
21\end{array}$ \\
\hline $\begin{array}{l}2.00 I-3.000 \\
300 I-4.000 \\
4.00 I-5.000\end{array}$ & $\begin{array}{l}86 \\
81 \\
82\end{array}$ & $\begin{array}{r}97 \\
96 \\
94\end{array}$ & $\begin{array}{l}85 \\
90 \\
87\end{array}$ & $\begin{array}{l}24 \\
21 \\
29\end{array}$ \\
\hline $\begin{array}{l}5.001-6.000 \\
6.001-7.000 \\
7.001-8.000 \\
8.001-9.000 \\
9.001-10.000\end{array}$ & $\begin{array}{l}80 \\
78 \\
73 \\
81 \\
65\end{array}$ & $\begin{array}{l}89 \\
85 \\
84 \\
79 \\
68\end{array}$ & $\begin{array}{l}88 \\
86 \\
84 \\
74 \\
63\end{array}$ & $\begin{array}{l}23 \\
32 \\
29 \\
36 \\
84\end{array}$ \\
\hline $\begin{array}{l}10.001-11.000 \\
11.001-13.000 \\
13.001-15.000\end{array}$ & $\begin{array}{l}66 \\
54 \\
36\end{array}$ & $\begin{array}{l}80 \\
66 \\
43\end{array}$ & $\begin{array}{l}65 \\
73 \\
50\end{array}$ & $\begin{array}{l}21 \\
22 \\
17\end{array}$ \\
\hline
\end{tabular}


Por roo Municipios de cada categoria, cuantos:

\begin{tabular}{|c|c|c|c|c|c|}
\hline $\begin{array}{c}\text { MUNICIPIOS } \\
\text { DE: } \\
\text { HABITANTES }\end{array}$ & $\underset{\text { tadero }}{\text { Sin ma- }}$ & $\begin{array}{l}\text { Sin cuarteles } \\
\text { Guardia civil }\end{array}$ & $\underset{\text { telégrafo }}{\operatorname{Sin}}$ & $\begin{array}{l}\text { Sin } \\
\text { teléfono }\end{array}$ & $\begin{array}{l}\text { Sin servicio } \\
\text { do incendios }\end{array}$ \\
\hline $\begin{array}{r}400 \\
401=\quad 600 \\
601-\quad 800 \\
801-1.000\end{array}$ & $\begin{array}{l}90 \\
79 \\
68 \\
61\end{array}$ & $\begin{array}{l}98 \\
94 \\
89 \\
85\end{array}$ & $\begin{array}{l}90 \\
81 \\
73 \\
72\end{array}$ & $\begin{array}{l}99 \\
99 \\
98 \\
96\end{array}$ & $\begin{array}{l}99 \\
98 \\
98 \\
95\end{array}$ \\
\hline $\begin{array}{l}1.001-1.200 \\
1.201-1.400 \\
1.401-1.600 \\
1.601-1.800 \\
1.801-2.000\end{array}$ & $\begin{array}{l}54 \\
49 \\
48 \\
44 \\
44\end{array}$ & $\begin{array}{l}74 \\
69 \\
61 \\
50 \\
46\end{array}$ & $\begin{array}{l}59 \\
58 \\
5^{8} \\
52 \\
51\end{array}$ & $\begin{array}{l}96 \\
95 \\
93 \\
90 \\
83\end{array}$ & $\begin{array}{l}97 \\
95 \\
91 \\
91 \\
92\end{array}$ \\
\hline $\begin{array}{l}2.001=3.000 \\
3.001=4.000 \\
4.001-5.000\end{array}$ & $\begin{array}{l}39 \\
29 \\
26\end{array}$ & $\begin{array}{l}41 \\
26 \\
24\end{array}$ & $\begin{array}{l}49 \\
36 \\
32\end{array}$ & $\begin{array}{l}84 \\
67 \\
66\end{array}$ & $\begin{array}{l}88 \\
82 \\
83\end{array}$ \\
\hline $\begin{array}{r}5.001-6.000 \\
6.001-7.000 \\
7.001-8.000 \\
8.001-9.000 \\
9.001-10.000\end{array}$ & $\begin{array}{l}30 \\
23 \\
20 \\
23 \\
12\end{array}$ & $\begin{array}{r}19 \\
21 \\
21 \\
23 \\
9\end{array}$ & $\begin{array}{l}29 \\
24 \\
25 \\
17 \\
14\end{array}$ & $\begin{array}{l}59 \\
47 \\
43 \\
44 \\
20\end{array}$ & $\begin{array}{l}83 \\
83 \\
77 \\
78 \\
63\end{array}$ \\
\hline $\begin{array}{l}10.001-11.000 \\
11.001-13.000 \\
13.001-15.000\end{array}$ & $\begin{array}{r}16 \\
12 \\
0\end{array}$ & $\begin{array}{r}12 \\
8 \\
0\end{array}$ & $\begin{array}{r}15 \\
11 \\
2\end{array}$ & $\begin{array}{r}22 \\
25 \\
5\end{array}$ & $\begin{array}{l}81 \\
69 \\
50\end{array}$ \\
\hline
\end{tabular}

Por 100 Municipios de cada categoria, cuantos:

\begin{tabular}{|c|c|c|c|c|c|}
\hline $\begin{array}{c}\text { MUNICIPIOS } \\
\text { DE: } \\
\text { HABITANTES }\end{array}$ & $\begin{array}{c}\text { Con } \\
\text { agua mala }\end{array}$ & $\begin{array}{l}\text { Con menos } \\
\text { de a litros } \\
\text { por habitante }\end{array}$ & $\begin{array}{l}\text { Sin fuentes } \\
\text { en el casco }\end{array}$ & $\begin{array}{c}\text { Sin red } \\
\text { domiciliaria }\end{array}$ & $\begin{array}{l}\text { Sin baños } \\
\text { públicos }\end{array}$ \\
\hline $\begin{array}{rr}- & 400 \\
401- & 600 \\
601- & 800 \\
801- & 1.000\end{array}$ & $\begin{array}{l}33 \\
36 \\
35 \\
35\end{array}$ & $\begin{array}{l}40 \\
43 \\
49 \\
53\end{array}$ & $\begin{array}{l}51 \\
44 \\
44 \\
39\end{array}$ & $\begin{array}{l}97 \\
95 \\
91 \\
92\end{array}$ & \\
\hline $\begin{array}{l}1.001-1.200 \\
1.201-1.400 \\
1.401-1.600 \\
1.601-1.800 \\
1.801-2.000\end{array}$ & $\begin{array}{l}35 \\
39 \\
36 \\
36 \\
35\end{array}$ & $\begin{array}{l}56 \\
65 \\
59 \\
63 \\
60\end{array}$ & $\begin{array}{l}40 \\
36 \\
32 \\
31 \\
28\end{array}$ & $\begin{array}{l}87 \\
86 \\
85 \\
82 \\
84\end{array}$ & \\
\hline $\begin{array}{l}2.001-3.000 \\
3.001-4.000 \\
4.001-5.000\end{array}$ & $\begin{array}{l}7 \\
9 \\
8\end{array}$ & $\begin{array}{l}41 \\
46 \\
43\end{array}$ & $\begin{array}{l}12 \\
13 \\
18\end{array}$ & $\begin{array}{l}77 \\
69 \\
73\end{array}$ & $\begin{array}{l}98 \\
96 \\
96\end{array}$ \\
\hline $\begin{array}{l}5.001-6.000 \\
6.001-7.000 \\
7.001-8.000 \\
8.001-9.000 \\
9.001-10.000\end{array}$ & $\begin{array}{l}7 \\
8 \\
9 \\
8 \\
5\end{array}$ & $\begin{array}{l}40 \\
39 \\
46 \\
36 \\
44\end{array}$ & $\begin{array}{r}15 \\
16 \\
11 \\
7 \\
7\end{array}$ & $\begin{array}{l}68 \\
70 \\
50 \\
66 \\
52\end{array}$ & $\begin{array}{l}94 \\
91 \\
92 \\
91 \\
80\end{array}$ \\
\hline $\begin{array}{l}10.001-11.000 \\
11.001-13.000 \\
13.001-15.000\end{array}$ & $\begin{array}{l}2 \\
9 \\
8\end{array}$ & $\begin{array}{l}49 \\
49 \\
29\end{array}$ & $\begin{array}{r}9 \\
15 \\
3\end{array}$ & $\begin{array}{l}47 \\
56 \\
25\end{array}$ & $\begin{array}{l}87 \\
83 \\
76\end{array}$ \\
\hline
\end{tabular}


Presupuesto de gastos

Tanto por 100 que representa cada Capitulo respecto del total

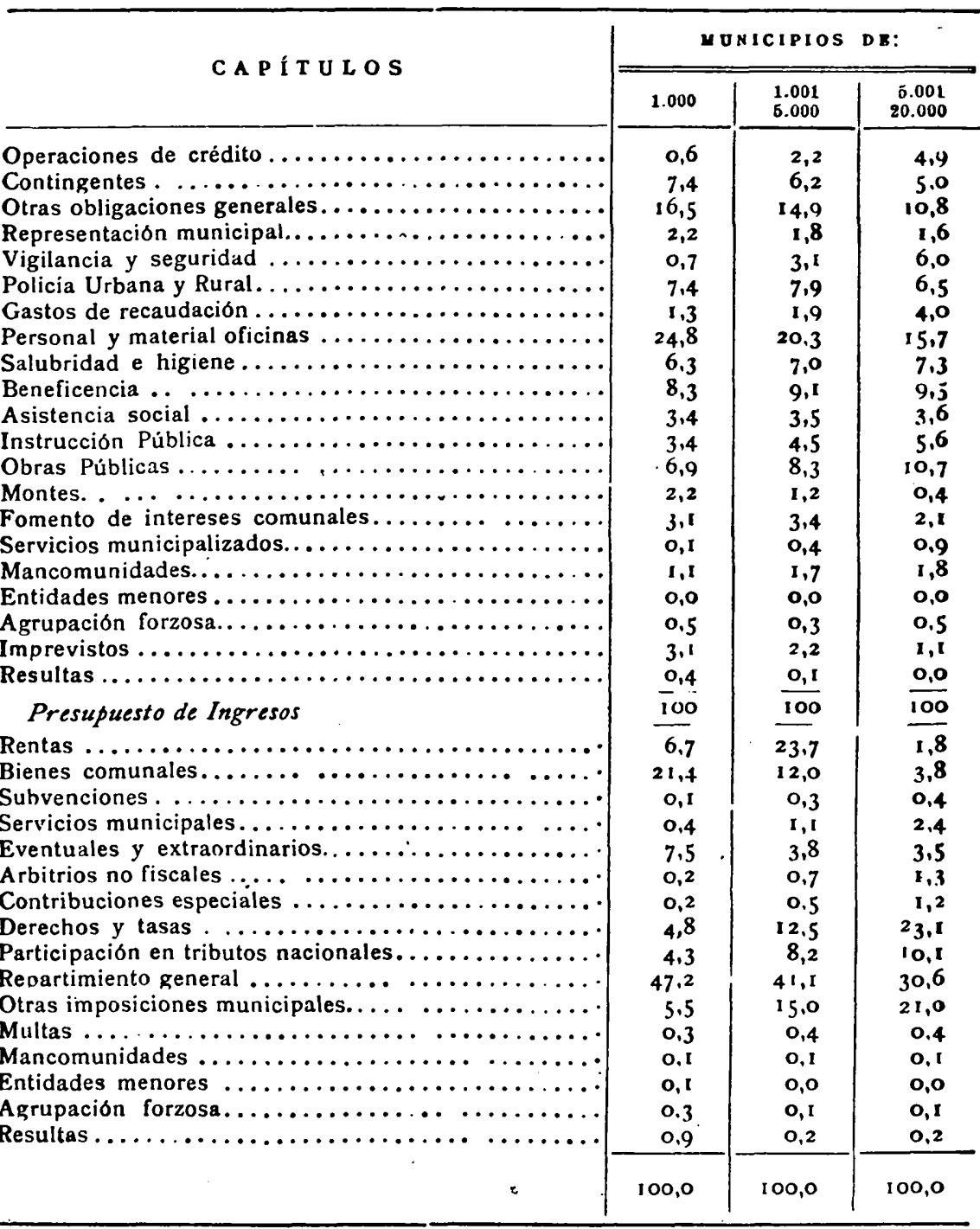

Javier. Ruiz Alimansa 